\title{
Computed Tomography Evaluation of Sinonasal Inverted Papilloma Characteristics with Origins of Tumors
}

\author{
Dilip Shah ${ }^{1}$, Prithviraj L. Jadhav ${ }^{1}$, Pradeep Shankarrao Patil' ${ }^{1}$ Ram Kumar Gupta ${ }^{1}$, Gaurav Verma ${ }^{1}$ \\ ${ }^{1}$ Associate Professor, Department of Radiodiagnosis, D.Y. Patil Medical College, Kolhapur-416003, India \\ Corresponding author: Dr. Prithviraj L. Jadhav, Associate Professor, Department of Radiodiagnosis, D.Y. Patil Medical College, \\ Kolhapur, India
}

DOI: http://dx.doi.org/10.21276/ijcmsr.2019.4.3.57

How to cite this article: Dilip Shah, Prithviraj L. Jadhav, Pradeep Shankarrao Patil, Ram Kumar Gupta, Gaurav Verma. Computed tomography evaluation of sinonasal inverted papilloma characteristics with origins of tumors. International Journal of Contemporary Medicine Surgery and Radiology. 2019;4(3):C258-C262.

\section{A B S T R A C T}

Introduction: Accuratefindings and excision of the originating site of a sinonasal inverted papilloma (SNIP) is important for decreasing tumor recurrence. Study aimed to evaluate the Computed Tomography (CT) characteristics of SNIP and to correlate these characteristics with the finding of the origins of tumors.

Material and Methods: Nasal cavity and paranasal sinus CT images of 38 patients were reviewed retrospectively to detect areas within which there was hyperostosis. We correlate SNIP characteristics with the detection of the origins of tumors described in the corresponding patient's medical records. We also assessed the CT features of hyperostosis according to the origin of tumors.

Result: Surgical evaluation of 29 lesions with hyperostosis in CT images revealed that 23 of these lesions coincided with the virtual origin of tumor. The CT-based determination of the positions of the areas of hyperostosis corresponded to the actual tumor origin in $79.3 \%$ of cases. Specifically, in cases with hyperostosis within the maxillary, frontal, posterior and sphenoid, ethmoid sinuses, areas of hyperostosis corresponded to the origin of tumor without detraction. In the evaluation of the CT characteristics of hyperostosis, two patterns of localized bone thickening were noted.

Conclusion: A high association between the origin of the SNIP and hyperostosis on CT might facilitate preoperative prediction of tumor origin by rhinologists and radiologists.

Keywords: Computed Tomography; Sinonasal Inverted Papilloma;Tumors; Hyperostosis

\section{INTRODUCTION}

Inverted papilloma is a rare tumor of the sinonasal cavity. Owing to a high percentage of recurrence and an association with squamous cell carcinoma, inverted papillomas are clinically destructive despite the pathologic benign nature of this type of tumor. Destructive surgical methods, such as medial maxillectomy via external incision, have been used to treat inverted papilloma. The accumulated experiences of endoscopic sinus surgical techniques, several surgeons have used relatively less invasive intranasal endoscopic approaches to resect Sinonasal Inverted Papilloma (SNIP). ${ }^{1}$ Exact preoperative evaluation with Computed Tomography (CT) and Magnetic Resonance Imaging (MRI) has also effect the trend toward the use of less invasive surgical approaches.

Currently, the radiologic characteristics of SNIP have been categorized..$^{2-8}$ In most of the previous findings, emphasis has been placed on the diagnostic significance of SNIP. Some studies have also focused on the identification of the extent of disease or tumor recurrence. To our knowledge, few studies have been carried out to determine whether image-based characteristics can be used to detect the origin of tumors, and the results of the studies were desperat., ${ }^{9,10}$ The identification of the origin of anSNIP is very important for the complete extermination of such tumors, because most recurrent tumors were localized to the same site as the primary tumors. ${ }^{4}$ Therefore, the aims of this study were to evaluate the CT characteristics of patients with SNIP and to correlate these characteristics with the detection of the origins of tumors.

\section{MATERIAL AND METHODS}

42 patients with pathologically diagnosed SNIP were treated at D.Y. Patil Medical College, Kolhapur from January 2015 to December 2018.

Recurrent lesions were identified in 11 of the patients, all of whom had already undergone either endoscopic surgery in other institutions. Because sinonasal bone thickening may occur postoperatively, the patients with a history of sinonasal surgery excluded. Four patients who had undergone MRI rather than a CT scan during preoperative evaluation were also excluded. Finally, CT images of 38 patients were reviewed retrospectively. There were $22(58 \%)$ males and 16 (42\%) females with a age range of 21-55 years (mean- 52.5 years).Squamous cell carcinoma was allied with SNIP in 3 patients.

Endoscopic tumor removal was performed in all patients, not only to find tissue for biopsy but also to control the tumor. 
An adjunctive transantral approach was also used in 11 patients. Tumor positive frontal sinuses were managed with an additional osteoplastic flap in 4 patients. Either single or multidetector row CT scanners (HiSpeed Advantage, LightSpeed QX/i,LightSpeed Ultra, or LightSpeed 16; GE Medical Systems, Milwaukee, Wis) were used because the patients were scanned over a duration of 5 years. Coronal and/or axial CT imaging was performed without administrating a resist agent. Contrast enhanced CT images, which were excluded from the image analysis in this study, were additionally obtained in some patients. Only coronal CT images were acquirable for 6 patients. The CT scans were acquired with $5 \mathrm{~mm}$ collimation and a table speed of $5 \mathrm{~mm} / \mathrm{s}$. The readjustment interval was $2.5-3.0 \mathrm{~mm}$.

The image analysis was performed by a radiologist who had experience in head and neck imaging and a rhinologist who had experience in endoscopic sinus surgery. The both of these analysts were do not know the medical history of the patients. The CT images of the nasal cavity and paranasal sinuses were examined with bone window setting, to detect areas within where there was hyperostosis. When evaluate hyperostosis in the paranasal sinuses (except for the ethmoid sinuses), we considered only hyperostosis to be a positive finding. The hyperostosis was defined as eccentric bone sclerosis and thickening that involved only a limited portion of the wall of a given paranasal sinus. We defined hyperostosis of the ethmoid sinus as thickening of the bone and sclerosis that involved only a part of the cells of the ethmoid sinuses. In cases that involved the lateral nasal wall, sclerosis of a certain structure, and localized bone thickening such as the uncinate process or a turbinate, was considered hyperostosis. We also evaluated the pattern of localised hyperostosis associated with the location of bony lesions.

We correlated the sites on the CT scans within which there was thickening of bone on CT with the origin of the tumors mentioned in the corresponding patient's medical records. The accurate origin of a tumor (area within, the bulk of the tumor was attached) was confirmed by the intraoperative endoscopic appraisal. At the time of surgery, histopathologic confirmation was used for suspicious mucosa and margins obtained from the sinonasal cavities.

\section{RESULTS}

The analysis of CT images revealed a total of 29 areas within which there was hyperostosis in 23 of 38 patients. The incidence of hyperostosis in patients with SNIP was $60.5 \%$. Most common site of tumor origin was the lateral nasal wall $17(44.7 \%)$. Tumors originated less frequently in the maxillary sinus $8(21.0 \%)$, anterior ethmoid sinus $5(13.1 \%)$, frontal sinus $3(7.9 \%)$, sphenoid sinus $3(7.9 \%)$, posterior ethmoid sinus $1(2.6 \%)$, and septum $1(2.6 \%)$. (Table 1$)$ There was no confirmation of bilateral tumor involvement in any patients. In 7 patients, tumors were attached in more than 1 compartment.

Surgical evaluation of 29 lesions associated with hyperostosis in CT images revealed that the location of hyperostosis for 23 of these lesions coincided with the actual tumor attachment site. Thus, the CT based determination of the positions of areas of hyperostosis corresponded to the actual

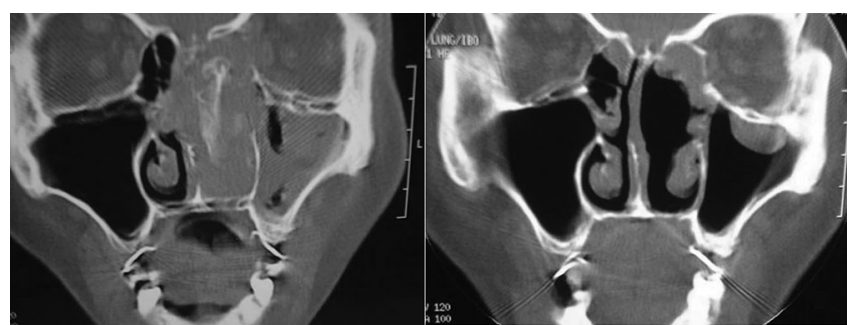

Figure-1: Pre-op and post op CT images (coronal view) and post of picture of same patient.

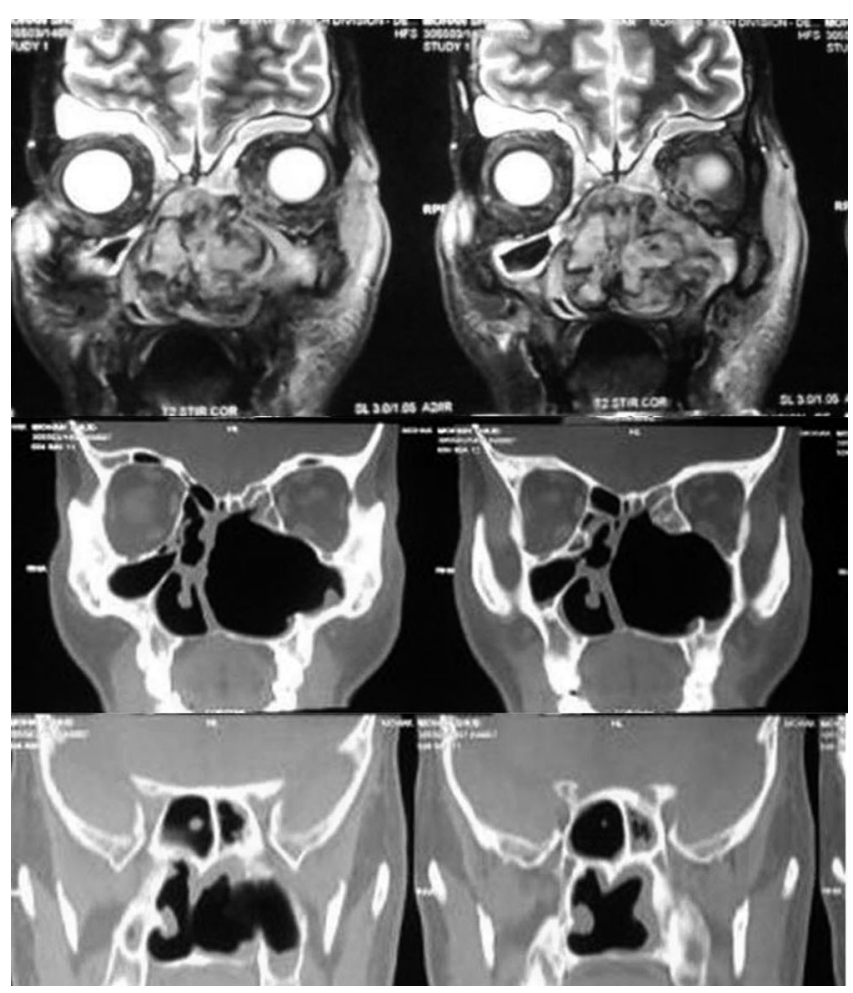

Figure-2: Pre-op MRI images and post-op CT images of a case of SNIP

(surgically determined) tumor origin in $79.3 \%$ of cases. In many cases, although tumor masses extended to the nasal cavity or adjacent sinuses, bone thickening was limited to the tumor origin as long as the origin did not extend to the nasal cavity or adjacent sinuses (Fig 1).

Areas of hyperostosis detected in CT scans were not associated with the actual site of tumor attachment in 3 cases. In these cases, areas of hyperostosis were observed in the ethmoid bulla (1 cases), uncinate process, superior turbinate, and middle turbinate. In all cases with hyperostosis within the nasal septum or maxillary, frontal, and sphenoid sinuses, these sites corresponded to the origin of the tumor.

This study revealed two patterns of localized bone thickening in cases with SNIP (Table 2). When hyperostosis involved the walls of the paranasal sinuses or the bony septum of the nasal cavity, the areas of hyperostosis were often times highly localized. The difference in thickness between the adjacent, unaffected bony wall was highly prominent and the hyperostotic area and resulted in a cone-shaped thickening of the bone was seen in 5 cases. (Fig 2). By resist, the thickness of plaque-like hyperostosis that involved the lateral nasal wall or anterior ethmoid sinus appeared to be 


\begin{tabular}{|l|c|c|c|}
\hline Origin of SNIP Confirmed by Surgery & Cases $(\mathbf{n}=\mathbf{3 8})$ & Hyperostosis on CT & $\begin{array}{c}\text { Coincidence of Hyperostotic } \\
\text { Area with Tumor Origin }\end{array}$ \\
\hline Lateral nasal wall & $17(44.7 \%)$ & 12 & 9 \\
\hline Maxillary sinus & $8(21.0 \%)$ & 7 & 7 \\
\hline Anterior ethmoid sinus & $5(13.1 \%)$ & 5 & 3 \\
\hline Frontal sinus & $3(7.9 \%)$ & 1 & 1 \\
\hline Sphenoid sinus & $3(7.9 \%)$ & 1 & 1 \\
\hline Posterior ethmoid sinus & $1(2.6 \%)$ & 1 & 1 \\
\hline Septum & $1(2.6 \%)$ & 1 \\
\hline *Data is given numbers (percentages); ${ }^{*}$ In 7 patients, tumor origin extended to more than one compartment of the sinonasal cavity. \\
\hline \multicolumn{2}{|r}{} \\
\hline
\end{tabular}

\begin{tabular}{|l|c|c|}
\hline Sites Having Areas of Hyperostosis & Cone-Shaped Hyperostosis on CT ( $\mathbf{n})$ & Plaque-Like Hyperostosis on CT (n) \\
\hline Lateral nasal wall & 0 & $11^{*}$ \\
\hline Maxillary sinus & 3 & $5^{\#}$ \\
\hline Anterior ethmoid sinus & 0 & 0 \\
\hline Frontal sinus & 1 & 1 \\
\hline Sphenoid sinus & 1 & 0 \\
\hline Posterior ethmoid sinus & 1 & 0 \\
\hline Septum & 1 & 0 \\
\hline$* *$ The lesions proved not to match with the origin of tumor; ${ }^{\#}$ The lesions proved not to match with the origin of tumor. \\
\hline \multicolumn{2}{|c|}{ Table-2: CT patterns of hyperostosis in SNIP } \\
\hline
\end{tabular}

relatively even compared with the cone-shaped pattern of hyperostosis (Fig 1). The plaque-like hyperostosis was seen irrespective of the spot of tumor origin. Although, coneshaped hyperostosis was seen only when the tumor originated from the walls of the paranasal sinuses except for anterior ethmoid sinuses.When the origins of SNIP were correlated with the areas of hyperostosis on CT, areas of cone-shaped hyperostosis eternally coincided with actual tumor origin, without exception. On the adverse, some of the plaque-like hyperostotic areas failed to predict the origin of SNIP.

\section{DISCUSSION}

SNIP is characterized by frequent reiteration and potential for malignancy. Therefore, accurate preoperative mapping of tumor locations is crucial to facilitate the effective resection of this type of tumor. MRI and CT can provide valuable information that can be used to delineate the extent of SNIP. MRI imaging can discriminate soft tissue tumors from adjacent areas of inflammation, and this imaging technique is superior to CT imaging for defining the location of a tumor. ${ }^{11}$ On the adverse, CT imaging is superior to MRI for evaluating changes in bone, such as hyperostosis, bone erosion, and bone destruction. Additional, utmostrhinologists, CT imaging in the coronal plane is the method of choice to create roadmaps in planning for endoscopic sinus surgery. ${ }^{12}$ Although, CT imaging has been reported to be of limited use in detecting the origin of tumors. Because of these limitations in the imaging of SNIP, endoscopic evaluation during surgery has been regarded as the only credible method for determining the areas in which tumors are attached. ${ }^{9,10}$

Changes in the bone that have been detected using CT scanning in patients with SNIP include intratumoral calcification, bowing, thinning, erosion, and sclerosis ${ }^{4,5,7}$ CT findings of bowing, bone thinning, and erosion might be due to an increase in the pressure exerted by the tumor as the tumor grows. So, it is unlikely that such changes in bone are associated with the origin site of the tumor. In our findings, there wereassociation betweenthe CT-based detection of areas within which there were sclerotic changes or endoscopic determination and hyperostosis of the tumor origin. However sclerotic changes of bone in the SNIP have been described previously, ${ }^{4,5,13}$ the relationship between these findings and the origin of tumors has not been examined in detail.

Generally, the origins of SNIP are localized to areas within the walls of the sinonasal cavity, despite the fact that these tumors usually have a large volume. SNIP is well known to exhibit a centrifugal growth pattern. ${ }^{3,14}$ The hyperostosis of the sinus wall is correlated with maxillary sinus tumor, endoscopic medial maxillectomy essential be planned preoperatively, because the tumor origin might be highly expected to be in the maxillary sinus. Lee et $\mathrm{a}^{10}$ highlighted that it is crucial to initially determine the location of the tumor infatuation site during surgery. So, preoperative identification of the tumor origin would be helpful in choosing a proper surgical approach. Preoperative CT-based detection of tumor origin may be especially auxiliary in case of frontal sinus disease, in which we often have difficulty in observing the origin of the tumor.

The mechanism that underlies the thickening of bone at the tumor origin of SNIP is unaware. We could notidentify the cause of the difference in the pattern of hyperostosis according to the tumor origin, either. Hyperostosis at the tumor indulgence site is a characteristic of meningiomas, and Pieper et $\mathrm{a}^{15}$ provided several possible elucidation of hyperostosis at the attachment site of meningiomas. Some of the elucidation might also apply to hyperostosis in SNIP, and they are tumor-induced vascular disruption within the underlying bone, irritation of the bone by the tumor, and so forth. The large bulky tumor volume associated with a relatively short 
area of attachment might result in hypervascularization at the attachment site, which could affect bone growth within the site. Because of mostly encountered substantial bleeding atsites there the cone-shaped hyperostotic bone has been removed during surgery in cases with SNIP that originated within the paranasal sinuses. Eisen et $\mathrm{a}^{16}$ proposed that an increase in vascularity due to SNIP may reason tinnitus.

Neogenesis or bone resorption is also associated with in chronic paranasal sinusitis, ${ }^{17,18}$ and some authors have reported that sclerotic bone changes in SNIP were related to accompanying or previous sinusitis. ${ }^{4,13}$ On the contrary, Lund and Lloyd ${ }^{5}$ reported the different patterns of hyperostosis between SNIP and chronic paranasal sinusitis. According to our study, hyperostosis in SNIP was usually localized and eccentric, which was apart from even, diffuse, and concentric pattern of sclerotic change in chronic paranasal sinusitis. Therefore, we coincide with Lund and Lloyd and believe that hyperostosis in SNIP might have nothing to do with chronic paranasal sinusitis.

SNIP generally originate from the lateral nasal wall or middle meatus and extend to adjacent paranasal sinuses or other nearby structures. ${ }^{19}$ Although, our series showed a relatively lower rate of tumor origin from the lateral nasal wall and the septum compared with other studies. Our institute is a tertiary care center; usually, relatively difficult cases for surgery are referred from other hospitals. Therefore, the dissent between our study and other studies might be a result of selection bias.

Because SNIP has a high possibility of recurrence and is locally aggressive, appropriate preoperative assessment of these tumors is crucial for successful treatment. Most reiteration occur at the same site at which the tumor originated and are frequently attributable to incomplete local resection. ${ }^{20,21}$ Furthermore, tumoral multiplicity has not been demonstrated determinately to be the cause of tumor recurrence. ${ }^{22}$ Therefore, to inhibit postoperative recurrence of tumors, precise preoperative identification of the tumor origin might be at least as important as detecting the extent of tumor invasion.

All patients with SNIP were not showed the CT finding of hyperostosis in our study. In those cases, without hyperostosis, we could not find any radiologic finding that helped in the detection of the origin of the tumor. However, when the finding of hyperostosis was seen, we could predict the origin of SNIP with a high possibility. Therefore, CTbased preoperative detection of tumor origin, although not possible in all cases, might facilitate the planning of surgery and prevent unnecessary resection of unaffected anatomic structures.

\section{CONCLUSION}

Hyperostosis visible in CT scans is associated repeatedly with SNIP. CT scans can be used to differentiate hyperostosis from diffuse bone thickening, which is usually associated with chronic paranasal sinusitis. Two distinct patterns of hyperostosis are associated with SNIP. The high association between the origin of the SNIP and hyperostosis could facilitate preoperative prediction of tumor origin by radiologists and rhinologists.

\section{REFERENCES}

1. Busquets JM, Hwang PH. Endoscopic resection of sinonasal inverted papilloma: a meta-analysis. Otolaryngol Head Neck Surg. 2006;134(1):476-82

2. Yousem DM, Fellows DW, Kennedy DW, et al. Inverted papilloma: evaluation with MR imaging. Radiology.1992; 185(3):501-05

3. Ojiri H, Ujita M, Tada S, et al. Potentially distinctive features of sinonasal inverted papilloma on MR imaging. AJR Am J Roentgenol. 2000; 175(5):465-68

4. Dammann F, Pereira P, Laniado M, et al. Inverted papilloma of the nasal cavity and the paranasal sinuses: using CT for primary diagnosis and follow-up. AJR Am J Roentgenol. 1999; 172(6):543-48

5. Lund VJ, Lloyd GA. Radiological changes associated with inverted papilloma of the nose and paranasal sinuses. Br J Radiol. 1984; 57(2):455-61

6. Maroldi R, Farina D, Palvarini L, et al. Magnetic resonance imaging findings of inverted papilloma: differential diagnosis with malignant sinonasaltumors. Am J Rhinol. 2004; 18(4):305-10

7. Woodruff WW, Vrabec DP. Inverted papilloma of the nasal vault and paranasal sinuses: spectrum of CT findings. AJR Am J Roentgenol. 1994; 162(1):419-23

8. Buchwald C, Nielsen LH, Ahlgren P, et al. Radiologic aspects of inverted papilloma. Eur J Radiol.1990; 10(4):134-39

9. Han JK, Smith TL, Loehrl T, et al. An evolution in the management of sinonasal inverting papilloma. Laryngoscope. 2001; 111(2):1395-400

10. Lee TJ, Huang SF, Huang CC. Tailored endoscopic surgery for the treatment of sinonasal inverted papilloma. Head Neck. 2004; 26(5):145-53

11. Savy L, Lloyd G, Lund VJ, et al. Optimum imaging for inverted papilloma. J Laryngol Otol. 2000; 114(4):89193

12. Mafee MF, Chow JM, Meyers R. Functional endoscopic sinus surgery: anatomy, CT screening, indications, and complications. AJR Am J Roentgenol. 1993; 160(1):735-44

13. Momose KJ, Weber AL, Goodman M, et al. Radiological aspects of inverted papilloma. Radiology.1980; 134(3):73-79

14. Michaels L. Benign mucosal tumors of the nose and paranasal sinuses. Semin Diagn Pathol.1996; 13(6):113-17

15. Pieper DR, Al-Mefty O, Hanada Y, et al. Hyperostosis associated with meningioma of the cranial base: secondary changes or tumor invasion. Neurosurgery. 1999; 44(3):742-47

16. Eisen MD, Buchmann L, Litman RS, et al. Inverted papilloma of the sphenoid sinus presenting with auditory symptoms: a report of two cases. Laryngoscope. 2002; 112(6):1197-200

17. Mirza N, Montone K, Sato Y, et al. Identification of $\mathrm{p} 53$ and human papilloma virus in Schneiderian papillomas. Laryngoscope. 1998; 108(1):497-501

18. Tovi F, Benharroch D, Gatot A, et al. Osteoblastic osteitis of the maxillary sinus. Laryngoscope.1992; 102(4):426-30

19. Phillips PP, Gustafson RO, Facer GW. The clinical 
behavior of inverting papilloma of the nose and paranasal sinuses: report of 112 cases and review of the literature. Laryngoscope. 1990; 100(5):463-69

20. Waitz G, Wigand ME. Results of endoscopic sinus surgery for the treatment of inverted papillomas. Laryngoscope. 1992; 102(1):917-22

21. McCary WS, Gross CW, Reibel JF, et al. Preliminary report: endoscopic versus external surgery in the management of inverting papilloma. Laryngoscope. 1994; 104(3):415-19

22. Nielsen PL, Buchwald C, Nielsen LH, et al. Inverted papilloma of the nasal cavity: pathological aspects in a follow-up study. Laryngoscope. 1991; 101(5):1094-101

Source of Support: Nil; Conflict of Interest: None

Submitted: 20-07-2019; Accepted: 19-08-2019; Published online: 25-09-2019 